\title{
Resident learning curve for minimal-access transforaminal lumbar interbody fusion in a military training program
}

\author{
Chris J. Neal, M.D., ${ }^{1}$ and Michael K. Rosner, M.D. ${ }^{2}$ \\ ${ }^{1}$ Division of Neurosurgery, National Naval Medical Center, Bethesda, Maryland; and ${ }^{2}$ Division of \\ Neurosurgery, Walter Reed Army Medical Center, Washington, DC
}

\begin{abstract}
Object. Minimal-access transforaminal lumbar interbody fusion (TLIF) has gained popularity as a method of achieving interbody fusion via a posterior-only approach with the aim of minimizing injury to adjacent tissue. While many studies have reported successful outcomes, questions remain regarding the potential learning curve for successfully completing this procedure. The goal of this study, based on a single resident's experience at the only Accreditation Council for Graduate Medical Education-approved neurosurgical training center in the US military, was to determine if there is in fact a significant learning curve in performing a minimal-access TLIF.

Methods. The authors retrospectively reviewed all minimal-access TLIFs performed by a single neurosurgical resident between July 2006 and January 2008. Minimal-access TLIFs were performed using a tubular retractor inserted via a muscle-dilating exposure to limit approach-related morbidity. The accuracy of screw placement and operative times were assessed.

Results. A single resident/attending team performed 28 minimal-access TLIF procedures. In total, 65 screws were placed at L-2 (1 screw), L-3 (2 screws), L-4 (18 screws), L-5 (27 screws), and S-1 (17 screws) from the resident's perspective. Postoperative CTs were reviewed to determine the accuracy of screw placement. An accuracy of 95.4\% (62 of 65) properly placed screws was noted on postoperative imaging. Two screws (at L-5 in the patient in Case 17 and at S-1 in the patient in Case 9) were lateral, and no revision was needed. One screw (at L-4 in Case 24) was $1 \mathrm{~mm}$ medial without symptoms or the need for revision. In evaluating the operative times, 2 deformity cases (Grade III spondylolisthesis) were excluded. The average operating time per level in the remaining 26 cases was 113.25 minutes. The average time per level for the first 13 cases was 121.2 minutes; the amount of time decreased to 105.3 minutes for the second group of 13 cases $(p=0.25)$.

Conclusions. In summary, minimal-access TLIF can be safely performed in a training environment without a significant complication rate due to the expected learning curve. (DOI: 10.3171/2010.1.FOCUS1011)
\end{abstract}

\begin{abstract}
KEY WoRds - minimal-access surgery resident education • learning curve
\end{abstract}

- transforaminal lumbar interbody fusion
A $\mathrm{s}$ the first few generations of residents graduate under the work-hour restrictions imposed by the Accreditation Council for Graduate Medical Education in 2003, competency in various procedures will come under increased scrutiny in the credentialing process. Further efforts must be made during the training period to maximize resident education and proficiency. One aspect of such efforts is to understand the number of procedures that must be performed before a trainee can be considered safe and competent. Comparing the ability to perform a task more efficiently over a period of time describes the term "learning curve." Although attempts have been made to understand the learning curves associated with various procedures, the neurosurgical and spine literature is lacking any such studies compared with what is found in other surgical fields..$^{1,2,5,6,8}$

Abbreviation used in this paper: TLIF = transforaminal lumbar interbody fusion.
There are certainly many variables associated with how an individual learns. In an attempt to understand this process we evaluated a single resident/attending team as the resident learned how to perform a minimal-access TLIF. This procedure has gained popularity as a method of achieving interbody fusion via a posterior-only approach with the aim of minimizing injury to adjacent tissue. ${ }^{9,15,18}$ While the authors of many studies have reported successful outcomes, questions remain regarding the process of learning to successfully and safely perform this procedure. The goal of this study, based on a single resident's experience, was to determine if there is a significant learning curve in performing a minimal-access TLIF.

\section{Methods}

We conducted a retrospective review of all minimalaccess TLIFs performed at a single center by a single resident/attending team between July 2006 and January 2008 . 


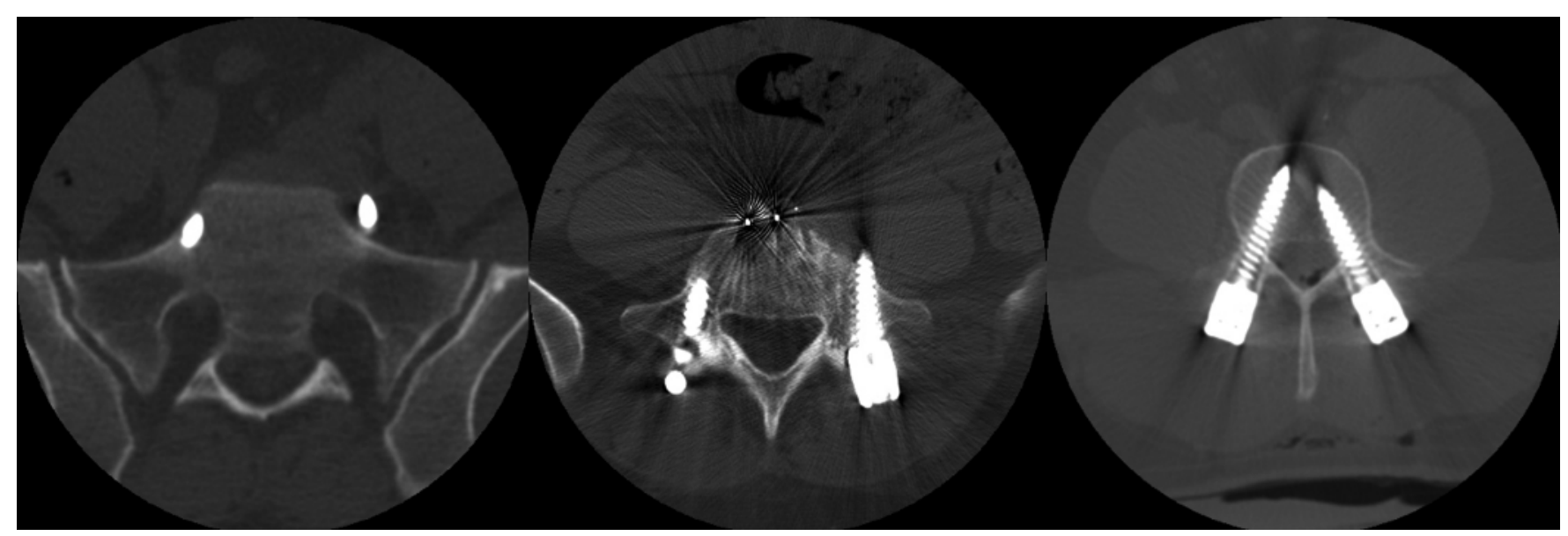

FIG. 1. Postoperative CT scans showing 2 screws (at S-1 in Case 9 and L-5 in Case 17) judged to have lateral screw placement and 1 screw (at L-4 in Case 24) with medial placement. These were clinically asymptomatic, and in none of these cases did the patient return to the operating room for screw revision.

The start date corresponded to the first minimal-access TLIF performed by the resident during his postgraduate Year 5. The resident had experience with pedicle screw placement and open TLIFs. The minimal-access TLIF was performed using a tubular retractor system (Quadrant, Medtronic) inserted via a muscle-dilating exposure to limit approach-related morbidity. Neuromonitoring was used in each case. In all cases, the resident worked on one side of the patient while the attending worked on the other side. The resident accessed the disc space and placed all screws and rods on his respective side. The disc space was identified after removing the superior aspect of the superior facet, making the superior aspect of the inferior pedicle flush with the disc space. The exiting nerve root and surrounding soft tissue were retracted superiorly from the inferior pedicle allowing for clear visualization of the disc. Pedicle screws were placed using direct visualization of the bony landmarks, specifically by identifying the junction of the pars and transverse process as well as the mammillary process when present. Placement was intraoperatively confirmed with fluoroscopy and pedicle screw stimulation. Each case was evaluated for the accuracy of screw placement and the operative time. The latter was obtained by reviewing our institution's computerized scheduling system in which the circulating nurse records the operative start and end times. The start time corresponded with skin incision, and the end time with incision closure. The accuracy of screw placement was judged according to the postoperative CT scans reviewed by the resident, attending, and a third independent observer. If the screw was not entirely contained within the pedicle, then it was considered to be out.

\section{Results}

Twenty-eight minimal-access TLIF cases that met the inclusion criteria were identified. There were 22 men and 6 women in the study with an average age of 42.7 years (range 18-80 years). The majority of the procedures were performed for degenerative disc disease. The resident placed 65 pedicle screws by using the featured ap- proach. One screw was placed at L-2, 2 at L-3, 18 at L-4, 27 at L-5, and 17 at S-1. Procedures were performed at L2-3 (1 case), L3-4 (1 case), L4-5 (9 cases), L5-S1 (9 cases), and L4-S1 (8 cases).

In a review of the postoperative $\mathrm{CT}$ scans, pedicle screw placement accuracy was determined to be $95.4 \%$, with 62 of the 65 pedicle screws placed entirely within the pedicle with an appropriate trajectory. Two screws (at L-5 in Case 17 and at S-1 in Case 9) were judged to be lateral, whereas 1 screw (at L-4 in Case 24) was medial (Fig. 1). There was no significant difference in pedicle screw accuracy between the first half of the patients compared with the second. Of the 33 pedicle screws placed in the first 14 patients, 32 (97\%) were considered accurate, compared with 30 of 32 pedicle screws $(93.8 \%)$ placed in the second group of 14 patients. None of the patients involved with pedicle breaches had postoperative deficits or symptoms related to screw placement, and none returned to the operating room for a revision.

In evaluating operative times, 2 cases with Grade III spondylolisthesis were excluded. The average operating time per level in the remaining 26 cases was 113.25 minutes. The average time per level for the first 13 cases was 121.2 minutes. This time decreased to 105.3 minutes for the second group of 13 cases $(p=0.25)$. The learning curve, based on operative time, was approximately 15 cases for this procedure (Fig. 2). The trend in increasing times toward the end of the study group was believed to be caused by the authors' use of this procedure for more advanced cases, as evident by the cases excluded in evaluating the operative times.

There were no intraoperative CSF leaks during the study period. Postoperatively, there was no new radiculopathy or weakness.

\section{Discussion}

Despite the fact that the current structure for neurosurgical postgraduate education has been around for decades, there is very little in the neurosurgery literature in regard to how a resident becomes competent and proficient at a particular procedure. While proficiency has 


\section{Learning Curve}

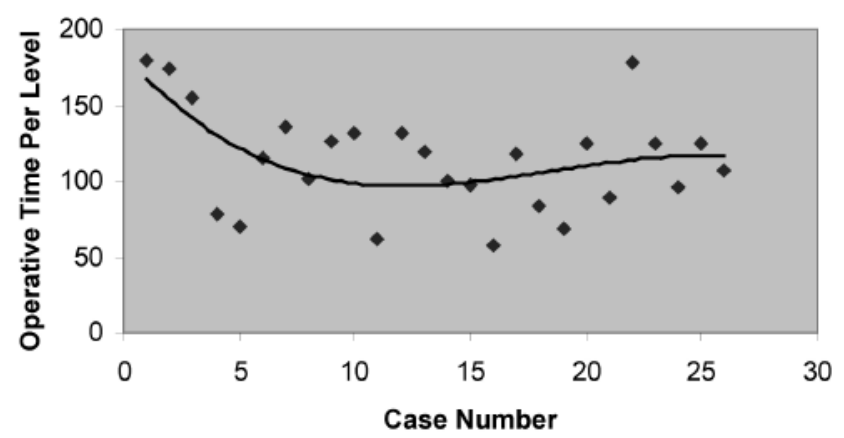

FIG. 2. Graph of the learning curve based on operative time per level. The level was approximately 15 cases. The trend in increasing times toward the end of the study group was believed to be due to more advanced cases.

always been important, it is particularly so now given that work-hour restrictions push residencies to become learning efficient. There is little doubt that an experienced surgeon tends to have shorter operating times and fewer complications. Such was the case in the study by Wiese et al. ${ }^{19}$ in which the authors found that the operative time was significantly shorter and the complication rate was significantly lower for an experienced surgeon compared with these measures for an inexperienced group performing lumbar microdiscectomies. This finding raises the question of when a trainee becomes experienced enough with a procedure so that he or she is competent. We believe that by constructing a learning curve, better insight into an individual's educational progress can be gained.

Applying the concept of a learning curve to medical and surgical education is not new, but its formal application is infrequent. Ramsay et al. ${ }^{16}$ conducted a study in which they searched the medical literature to ascertain how learning curves are evaluated. In searching multiple databases, they found 4571 abstracts that contained the phrase "learning curve." Among the studies represented by these abstracts, only $272(6 \%)$ had evaluated the learning curve through statistical methods. Authors often rely on anecdotal evidence and descriptive terms such as "steep" or "long" instead of scientific evaluation. ${ }^{4,10}$

Several articles in the literature address the learning curve associated with spinal procedures. In 2005 Nowitzke $^{14}$ evaluated the first 35 microendoscopic discectomies performed for lumbar radiculopathy. Surgical time, conversion to an open procedure, complication rates, and surgeon comfort were taken into account. The author concluded that it takes approximately 30 cases to become proficient with the procedure. In a similar paper, McLoughlin and Fourney ${ }^{13}$ looked at a single surgeon's experience with the first 52 consecutively performed minimally invasive microdiscectomies using a tubular retractor system. Using mainly operative time to analyze their learning curve, these authors concluded that after approximately 15 cases, a steady state was reached. These studies are similar to our work in that the authors evaluated the learning curve of adapting a minimally invasive technique to a procedure they had previously performed by an open surgical method. Our study differs in that it is focused on a resident's learning curve instead of an attending surgeon's.

Some authors believe that operative time is not a good means of evaluating a surgeon's learning curve and that other factors should be more heavily weighted. Chen et al. ${ }^{3}$ analyzed 287 consecutive laparoscopic colorectal resections and found that operative times did not decrease over time. In fact, the surgeon with the shorter operative times had higher complication and readmission rates. Chen and colleagues concluded that learning curves for laparoscopic colorectal surgery should be based on complication and readmission rates as opposed to operative times and conversion rates. If the goal is to determine how many cases it takes a surgeon to learn how to perform a procedure proficiently as well as safely, then the authors' point is well taken in that multiple factors, not just operative times, should be considered. Evaluating complications, readmissions, patient satisfaction, and long-term outcome is crucial in obtaining a true understanding of when a surgeon has truly mastered the procedure. Speed in the operating room does not always correlate with successful patient outcomes.

The other end point in this study was pedicle screw accuracy. In comparing our results with those in the literature, we noted that a $95.4 \%$ accuracy rate is at or above the rate reported in most series. ${ }^{7,12,17}$ In 2005 Kosmopoulos and Schizas ${ }^{11}$ published a meta-analysis of the literature on pedicle screw placement. Subgroup analysis revealed 7 studies that evaluated in vivo placement of lumbar pedicle screws without the aid of navigation. Among the 1674 pedicle screws described in these studies, the mean placement accuracy rate was $87.3 \%$ with a range of $60-97.5 \%$. The weakness in comparing our accuracy rate with that in this meta-analysis is the lack of consistency in how accuracy is defined.

\section{Conclusions}

Learning to perform a minimal-access TLIF can be done safely in a training environment without a significant complication rate. Pedicle screw placement accuracy was not diminished in learning the new approach. In this study, it took approximately 15 cases for the learning curve to plateau based on operative times. While we have yet to apply learning curves for all resident cases, we believe, based on these results, that this relatively straightforward measurement can be easily applied to track resident progress for core training cases. Because of the nuances of each training program, learning curves should be individualized to the institution with which they are associated. For future studies, we are currently planning to compare larger numbers of trainees at different experience levels and to assess long-term outcome and the affect the learning curve has on it.

\section{Disclosure}

The authors report no conflict of interest concerning the materials or methods used in this study or the findings specified in this paper.

The opinions or assertions contained herein are the private views of the authors and are not to be construed as official or as 


\section{J. Neal and M. K. Rosner}

reflecting the views of the US Army, US Navy, or US Department of Defense.

Author contributions to the study and manuscript preparation include the following. Conception and design: both authors. Acquisition of data: CJ Neal. Analysis and interpretation of data: both authors. Drafting the article: both authors. Critically revising the article: both authors. Reviewed final version of the manuscript and approved it for submission: MK Rosner. Statistical analysis: CJ Neal.

\section{References}

1. Ascher-Walsh CJ, Capes T: An evaluation of the resident learning curve in performing laparoscopic supracervical hysterectomies as compared with patient outcome: five-year experience. J Minim Invasive Gynecol 14:719-723, 2007

2. Cagir B, Rangraj M, Maffuci L, Herz BL: The learning curve for laparoscopic cholecystectomy. J Laparoendosc Surg 4: 419-427, 1994

3. Chen W, Sailhamer E, Berger DL, Rattner DW: Operative time is a poor surrogate for the learning curve in laparoscopic colorectal surgery. Surg Endosc 21:238-243, 2007

4. Eck JC, Hodges S, Humphreys SC: Minimally invasive lumbar spinal fusion. J Am Acad Orthop Surg 15:321-329, 2007

5. Forbes TL, DeRose G, Kribs SW, Harris KA: Cumulative sum failure analysis of the learning curve with endovascular abdominal aortic aneurysm repair. J Vasc Surg 39:102-108, 2004

6. Fraser SA, Feldman LS, Stanbridge D, Fried GM: Characterizing the learning curve for a basic laparoscopic drill. Surg Endosc 19:1572-1578, 2005

7. Grauer JN, Vaccaro AR, Brusovanik G, Girardi FP, Silveri CP, Cammisa FP, et al: Evaluation of a novel pedicle probe for the placement of thoracic and lumbosacral pedicle screws. J Spinal Disord Tech 17:492-497, 2004

8. Hogle NJ, Briggs WM, Fowler DL: Documenting a learning curve and test-retest reliability of two tasks on a virtual reality training simulator in laparoscopic surgery. J Surg Educ 64:424-430, 2007

9. Holly LT, Schwender JD, Rouben DP, Foley KT: Minimally invasive transforaminal lumbar interbody fusion: indications, technique, and complications. Neurosurg Focus 20(3):E6, 2006
10. Hoogland T, van den Brekel-Dijkstra K, Schubert M, Miklitz B: Endoscopic transforaminal discectomy for recurrent lumbar disc herniation: a prospective, cohort evaluation of 262 consecutive cases. Spine 33:973-978, 2008

11. Kosmopoulos V, Schizas C: Pedicle screw placement accuracy: a meta-analysis. Spine 32:E111-E120, 2007

12. Laine T, Lund T, Ylikoski M, Lohikoski J, Schlenzka D: Accuracy of pedicle screw insertion with and without computer assistance: a randomised controlled clinical study in 100 consecutive patients. Eur Spine J 9:235-240, 2000

13. McLoughlin GS, Fourney DR: The learning curve of minimally-invasive lumbar microdiscectomy. Can J Neurol Sci 35:75-78, 2008

14. Nowitzke AM: Assessment of the learning curve for lumbar microendoscopic discectomy. Neurosurgery 56:755-762, 2005

15. Ozgur BM, Yoo K, Rodriguez G, Taylor WR: Minimally-invasive technique for transforaminal lumbar interbody fusion (TLIF). Eur Spine J 14:887-894, 2005

16. Ramsay CR, Grant AM, Wallace SA, Garthwaite PH, Monk AF, Russell IT: Assessment of the learning curve in health technologies. A systematic review. Int J Technol Assess Health Care 16:1095-1108, 2000

17. Schulze CJ, Munzinger E, Weber U: Clinical relevance of accuracy of pedicle screw placement. A computed tomographicsupported analysis. Spine 23:2215-2221, 1998

18. Schwender JD, Holly LT, Rouben DP, Foley KT: Minimally invasive transforaminal lumbar interbody fusion (TLIF): technical feasibility and initial results. J Spinal Disord Tech 18 (Suppl):S1-S6, 2005

19. Wiese M, Kramer J, Bernsmann K, Ernst Willburger R: The related outcome and complication rate in primary lumbar microscopic disc surgery depending on the surgeon's experience: comparative studies. Spine J 4:550-556, 2004

Manuscript submitted January 7, 2010.

Accepted January 20, 2010.

Address correspondence to: Chris J. Neal, M.D., National Naval Medical Center, 8901 Rockville Pike, Bethesda, Maryland 20889. email: chrisjneal@verizon.net. 